Technological University Dublin ARROW@TU Dublin

\title{
Fundamental Efficiency Limit of Passive Non-Magnetic Self- Interference Suppression in Full-Duplex Radios
}

\author{
Adam Narbudowicz \\ Technological University Dublin, adam.narbudowicz@mydit.ie \\ Giuseppe Ruvio \\ National University of Ireland, Galway \\ Max Ammann \\ Technological University Dublin, max.ammann@tudublin.ie
}

See next page for additional authors

Follow this and additional works at: https://arrow.tudublin.ie/ahfrccon

Part of the Electrical and Computer Engineering Commons

\section{Recommended Citation}

A. Narbudowicz, G. Ruvio, M. J. Ammann and N. Marchetti, "Fundamental Efficiency Limit of Passive NonMagnetic Self-Interference Suppression in Full-Duplex Radios," 2018 IEEE International Symposium on Antennas and Propagation \& USNC/URSI National Radio Science Meeting, 2018, pp. 741-742, doi: 10.1109/APUSNCURSINRSM.2018.8609456.

This Conference Paper is brought to you for free and open access by the Antenna \& High Frequency Research Centre at ARROW@TU Dublin. It has been accepted for inclusion in Conference Papers by an authorized administrator of ARROW@TU Dublin. For more information, please contact arrow.admin@tudublin.ie, aisling.coyne@tudublin.ie,gerard.connolly@tudublin.ie.

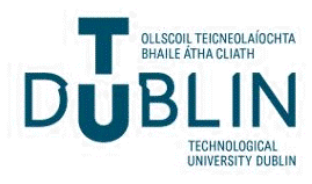


Authors

Adam Narbudowicz, Giuseppe Ruvio, Max Ammann, and Nicola Marchetti

This conference paper is available at ARROW@TU Dublin: https://arrow.tudublin.ie/ahfrccon/51 


\title{
Fundamental Efficiency Limit of Passive Non-Magnetic Self-Interference Suppression in Full-Duplex Radios
}

\author{
Adam Narbudowicz ${ }^{1,3}$, Giuseppe Ruvio ${ }^{1,2}$, Max J. Ammann ${ }^{1}$ and Nicola Marchetti ${ }^{3}$ \\ ${ }^{1}$ Antenna \& High Frequency Research Centre \\ ${ }^{2}$ College of Engineering and Informatics \\ Dublin Institute of Technology \\ Dublin, Ireland \\ \{adam.narbudowicz; max.ammann\}@dit.ie \\ National University of Ireland Galway \\ Galway, Ireland \\ giuseppe.ruvio@nuigalway.ie \\ ${ }^{3}$ CONNECT Centre \\ Trinity College Dublin, the University of Dublin \\ Dublin, Ireland \\ nicola.marchetti@tcd.ie
}

\begin{abstract}
Passive non-magnetic self-interference suppression circuits are currently used as first stages of complex full-duplex radio systems that allow simultaneous reception and transmission within the same channel. This paper introduces the upper efficiency limit for such circuitry. It is demonstrated that for these devices it is not feasible to have simultaneously high efficiency, good transmitter-to-receiver isolation and same propagation channel for transmission and reception. More specifically, the maximum efficiency for most typical case cannot exceed $50 \%$ if good isolation is obtained.
\end{abstract}

Keywords-full-duplex radio; simultaneous transmission and reception (STAR); efficiency; antenna; antenna feed

\section{INTRODUCTION}

Full-duplex radios, also known as Simultaneous Transmit and Receive (STAR) system, are attracting significant attention due to recent advancements in passive self-interference suppression [1]. Historically, transmitters and receivers operating within single device in full-duplex mode were separated either by bulky magnetic circulators or large antenna separation. Both approaches are not implementable within miniaturized radio devices, e.g. for Internet of Things or wireless sensor applications.

As part of the effort to realize miniaturized full-duplex radios, some attention was given to passive self-interference suppression by antenna and antenna feed circuitry [2-4]. This is typically the first stage of complex circuitry, which aims to reduce self-interference to suitable level by using both analogue and digital techniques on subsequent stages.

This paper outlines basic physical limitations of the antenna and linear microwave circuitry for self-interference suppression. It is demonstrated that transmission through the same propagation channel with good transmitter-to-receiver isolation necessitates decreased efficiency of the system, regardless of the antennas system used. For most typical cases the upper efficiency limit is $50 \%$, which is consistent with state-of-the-art passive non-magnetic self-interference suppression proposed in the literature [4].

\section{FULL-DUPLEX VS. MIMO}

In order for the full-duplex radio to operate efficiently, transmission and reception need to occur within the same channel. This requirement is especially critical for cognitive radio applications, where a timely detection of the active primary user in the same channel is of key importance. From an antenna point of view, it makes a clear distinction between Full-duplex and MIMO systems: in the latter antennas are designed to use multiple orthogonal channels in order to maximize communication throughput. In terms of antenna performance, it is usually evaluated by Envelope Correlation Coefficient (ECC)

It is proposed in [4] that the same metric can be beneficial to analyse antennas dedicated to full-duplex applications:

$\rho_{E C C}=\frac{\iint P_{R X}(\theta, \phi) P_{T X}^{*}(\theta, \phi) d \Omega}{\sqrt{\iint P_{R X}(\theta, \phi) P_{R X}^{*}(\theta, \phi) d \Omega \iint P_{T X}(\theta, \phi) P_{T X}^{*}(\theta, \phi) d \Omega}}$

where $P_{R X}$ is the received radiation pattern and $P_{T X}$ is the transmit radiation pattern. Both patterns include polarimetric information which is not shown for brevity.

Since in full-duplex radio one requires transmission and reception within the same channel, this is guaranteed when $\mathrm{ECC}=1$. It therefore puts design criteria for MIMO antennas on the opposite side to Full-duplex antennas. However, from a system perspective, both allow to increase communication capacity. 


\section{EFFICIENCY}

The requirement of using the same channel for transmission and reception has significant consequences for the design of passive non-magnetic self-interference suppression circuits. Such circuits are usually the first stage to reduce selfinterference that leaks energy from transmitter to receiver.

In [5] Hallbjorner proposed a formula that - for passive linear circuits - binds ECC with S-parameters and efficiencies:

$$
\begin{aligned}
& 0=\frac{S_{T T} S_{T R}^{*}+S_{R T} S_{R R}^{*}}{\sqrt{\left(1-\left|S_{T T}\right|^{2}-\left|S_{R T}\right|^{2}\right)\left(1-\left|S_{R R}\right|^{2}-\left|S_{T R}\right|^{2}\right)}}+\rho_{E C C} \sqrt{\eta_{R} \eta_{T}}+ \\
& +\rho_{\text {loss }} \sqrt{\left(1-\eta_{T}\right)\left(1-\eta_{R}\right)}
\end{aligned}
$$

where $\eta_{T}$ and $\eta_{R}$ denote efficiency of transmitter and receiver antenna and feeds respectively, while $\rho_{\text {loss }}$ is correlation of losses occurring in the system.

In order to provide good self-interference suppression, the system should exhibit good isolation, i.e. lowest possible $S_{R T}$. If this is satisfied, the first term in (2) equals zero. Both efficiencies take values from 0 to 1 . Exact measurement of the

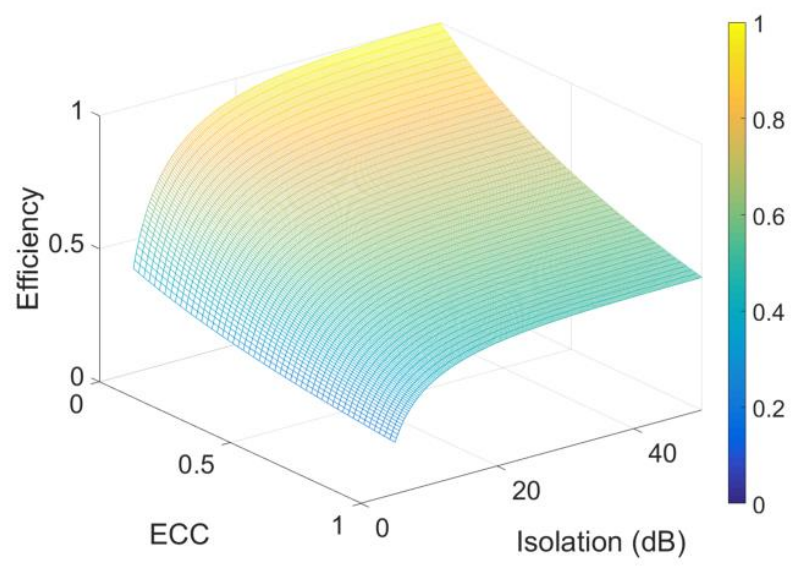

a)

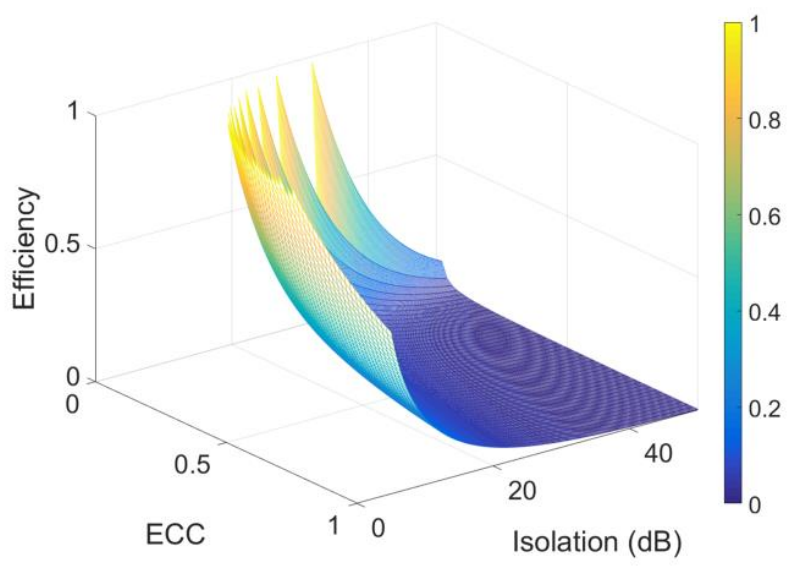

b)

Fig. 1. Efficiency as a function of channel correlation and isolation for: a) $\rho_{\text {loss }}=-1$; b) $\rho_{\text {loss }}=0$. correlation of losses $\rho_{\text {loss }}$ is challenging, however its amplitude cannot exceed one. Proper operation of full-duplex radio requires that transmission and reception occur in the same channel, which corresponds to $\rho_{E C C}=1$. Applying those values to (2) and assuming perfect isolation $\left(S_{R T}=-\infty \mathrm{dB}\right)$ and same channel for transmission and reception $\left(\rho_{E C C}=1\right)$, we can derive the upper limit on the efficiencies of the antenna and passive self-interference suppression circuitry:

$$
\frac{\sqrt{\eta_{T} \eta_{R}}}{\sqrt{\left(1-\eta_{T}\right)\left(1-\eta_{R}\right)}} \leq 1
$$

The above limit applies only to the passive non-magnetic suppression, which is usually the first stage of the full-duplex radio implementation. If $\eta_{T}=\eta_{R}$ the maximum achievable efficiency is $50 \%$.

Since passive suppression is only the first stage of fullduplex radio systems, one may choose to reduce isolation as it can be compensated at later stages. Similarly, for some applications one may allow small discrepancy between transmit and receive channels, which will results in $\rho_{E C C}<1$. Efficiency calculated for such cases is shown in Fig 1 for two most significant scenarios: $\rho_{\text {loss }}=-1$ (Fig. 1a) and $\rho_{\text {loss }}=0$ (Fig. 1b). Calculations assume $\mathrm{S}_{\mathrm{TT}}=\mathrm{S}_{\mathrm{RR}}=-10 \mathrm{~dB}$ and $\eta_{T}=\eta_{R}$.

\section{CONCLUSIONS}

The paper quantifies an upper efficiency limit for antennas and passive non-magnetic microwave circuitry used for selfinterference suppression in full-duplex radios. It is demonstrated that decreased efficiency is the price that needs to be paid for good transmitter-to-receiver isolation if same radio channel is used for transmission and reception.

\section{ACKNOWLEDGMENT}

This project has received funding from the European Union's Horizon 2020 research and innovation programme under the Marie Skłodowska-Curie grant agreement No 713567 and from Science Foundation Ireland (SFI) under Grant Number 13/RC/2077.

\section{REFERENCES}

[1] N. Reiskarimian, and H. Krishnaswamy, "Magnetic-free non-reciprocity based on staggered commutation", Nature Communications, vol. 7, Apr. 2016.

[2] J. I. Choi, M. Jain, K. Srinivasan, P. Levis, and S. Katti, "Achieving single channel, full duplex wireless communication", in Proc. MobiCom '10, $16^{\text {th }}$ annual international conference on mobile computing and networking, Chicago, IL, USA, Sept. 2010.

[3] L. Laughlin, C. Zhang, M. A. Beach, K. A. Morris, and J. L. Haine, "Passive and Active Electrical Balance Duplexers", IEEE Transactions on Circuits and Systems II: Express Briefs, vol. 63, no. 1, pp. 94-98, Jan. 2016.

[4] A. Narbudowicz, G. Ruvio, and M.J. Ammann, "Passive SelfInterference Suppression for Single Channel Full-Duplex Operation", IEEE Wireless Communications Magazine, In Press.

[5] P. Hallbjorner, "The significance of radiation efficiencies when using SParameters to calculate the received signal correlation from two antennas", IEEE Antennas and Wireless Propagation Letters, vol. 4, pp. $97-99,2005$ 